\title{
SERIAL SEARCH CODE ACQUISITION OF FHSS SYSTEM
}

\author{
MUHANNED AL-RAWI ${ }^{1, \mathrm{a}}$, MUAAYED AL-RAWI 2 , b \\ ${ }^{1}$ University of Ibb, Yemen \\ ${ }^{2}$ University of AL-Mustansiryia, Iraq \\ a'E-mail: muhrawi@yahoo.com \\ bE-mail: muaayed@yahoo.com
}

Frequency hopping spread spectrum (FHSS) communication systems offer significant performance advantages in view of their low probability of intercept, improved performance in multipath fading environments and their ability to avoid interference by hopping into low interference frequency channels. For the transmitted sequence to be correctly received and demodulated, the frequency hop sequence used at the receiver should be similar to that employed in the transmitter. Code acquisition in frequency hopping attempts to address this problem by providing a frequency hop pattern at the receiver that is nearly identical to that used at the transmitter. Code acquisition brings the alignment between the transmitter and the receiver hop pattern to at least one hop period. In this paper, the serial search code acquisition, which is the first step of synchronization, is studied for frequency hopping spread spectrum (HFSS) system over Rayleigh fading channel. Simulation results show the effect of fading channel on the code acquisition.

Keywords: FHSS system, serial search code acquisition, Rayleigh fading channel, FSK modem

\section{Introduction}

A spread spectrum communication system is a communication system in which, broadly speaking, the transmission bandwidth used is much greater than the actual bandwidth that is required to transmit the information $[1,2]$.

Unlike standard modulation schemes such as frequency modulation, that also cause the spectrum of the modulated message signal to expand in excess of the information rate, spread spectrum systems employ spreading signals that are not related to the information $[3,4]$.

The spreading sequences that are deployed, appear to be random and are for this reason referred to as pseudorandom (PN) sequences. These sequences are used at the transmitter to spread the message signal and at the receiver to recover the transmitted message [5].

Essentially, for a system to be defined as a spread spectrum system it has to meet a set of conditions. The following conditions provide a rationale for determin- ing whether or not a system is a spread spectrum system [6]:

- The message signal being transmitted must eventually occupy a bandwidth way larger than the minimum bandwidth that is necessary to send the information.

- The spreading of the signal should be accomplished by means of a spreading signal which is unrelated to the message data.

- The process of dispreading, i.e. recovering the original data at the receiver is achieved by correlating the received spreading signal with a synchronized replica of the spreading signal that was initially used to spread the information at the transmitter.

There are several types of spread spectrum systems, but this paper focuses on frequency hopping spread spectrum (FHSS) system. An important point in FHSS system is code synchronization which involves two steps, namely; code acquisition, and code tracking. The code acquisition phase involves bringing

Open Access statement. This is an open-access article distributed under the terms of the Creative Commons AttributionNonCommercial 4.0 International License (https://creativecommons.org/licenses/by-nc/4.0/), which permits unrestricted use, distribution, and reproduction in any medium for non-commercial purposes, provided the original author and source are credited, a link to the CC License is provided, and changes - if any - are indicated. 
the PN sequence to at least within a time period of the $\mathrm{PN}$ sequence used to generate the carrier frequency at the receiver. Code tracking involves further refining the acquired PN sequence improving its accuracy to less than half the time period of the transmitted signal $[7,8]$.

\section{System analysis and design}

Code acquisition in a FHSS is simulated using the MATLAB Simulink simulation software. Appropriate Simulink block sets are used and their parameters varied to conform to design specifications. For generation of the hopping codes, PN sequences are used due to their correlation properties that allow for easier acquisition. For simulation purposes the Rayleigh fading channel is simulated. The Simulink block diagram in Fig. 1 outlines the complete design.

A few of the various design aspects of the common block parameters are designed in the next few paragraphs. The rationale for arriving at each of the design parameters is also discussed.

\subsection{Bernoulli binary generator}

The Bernoulli binary generator is chosen as the simulation data source since it outputs a random sequence of 0 and 1 bit sequences. The probability of getting a 0 and a 1 are all set to be equal, i.e. both have a probability of 0.5 .

The bit rate for the Bernoulli binary generator is set at 10,000 bits per second. Before the gener- ated Bernoulli sequence is mixed with the carrier frequency generated through the 8-Frequency-Shift-Keying (FSK) modulator, it is first 2-FSK modulated.

At the receiver, a 2-FSK demodulator is used for demodulation. If the demodulation process is effective, an exact replica of what has been transmitted should be received.

\subsection{PN sequence}

A 4-stage LFSR with a PN sequence length of 15 is used. The LFSR used is a maximal sequence LFSR. Similar PN sequence generators are used at both the transmitter and the receiver. The output bit rate for both PN sequence generators is set at 90,000 bits per second.

The generated bits from the PN sequence generator are then clustered into groups of 3 bits and a bit to integer converter is used to generate eight possible integer values ranging from 0 to 7 . The output of the bit to integer converter is then fed to an 8-FSK modulator which then outputs the different carrier frequencies that are used to effect frequency hopping.

Since the output bit rate from the PN sequence generator is 90,000 bits per second and they are grouped into clusters of 3 , there are 30,000 clusters generated per second. Therefore, ideally, the output frequency of the 8-FSK modulator varies 3 times per symbol of the data sequence generated by the Bernoulli binary generator.

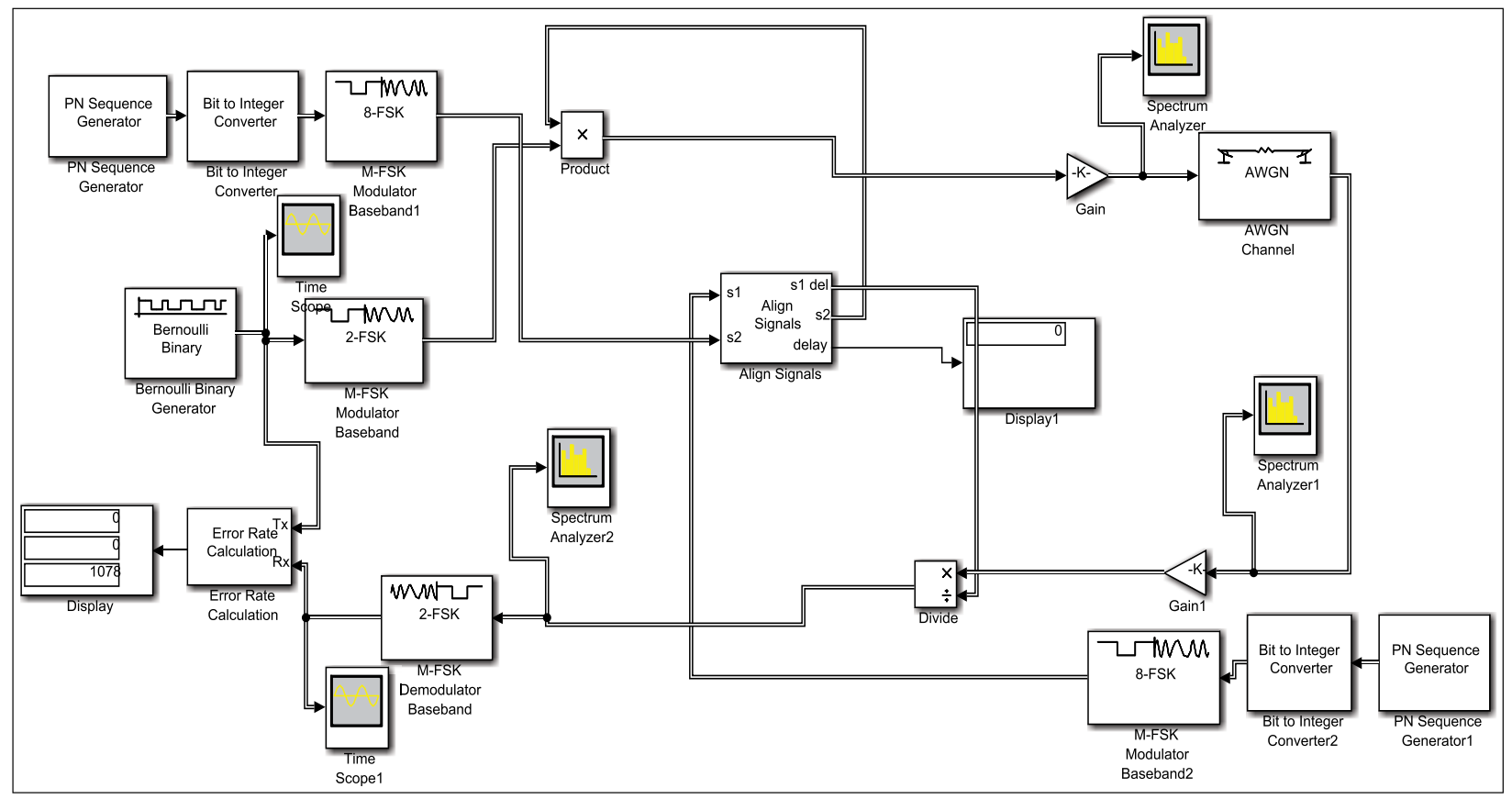

Fig. 1. Simulation block for serial search acquisition for a FHSS system 


\subsection{8-FSK modulator}

The 8-FSK modulator is set to have a carrier frequency separation of $100 \mathrm{kHz}$. The eight possible carrier frequencies therefore range from $100 \mathrm{kHz}$ to $800 \mathrm{kHz}$.

\subsection{Align signals block}

The align signals block is the block that performs the serial search acquisition. The align signals block compares two signals by correlating them and then adjusts the signal to be adjusted based on the correlation peak. PN sequences offer a lot of demodulation ease since they have specific correlation peaks.

In the design highlighted above, before the PN sequence that is used at the transmitter is transmitted to the align signal block at the receiver, it is first 8-FSK modulated. The PN sequence at the receiver is also 8-FSK modulated and after this the PN sequence at the receiver is aligned to that received from the transmitter. The align signal block periodically delays the PN sequence at the receiver until it is aligned to the one at the receiver.

\subsection{Rayleigh fading channel}

In the typical propagation of electromagnetic signals different paths may exist. The direct path from a transmitter to a receiver is known as the line of sight path. The other paths that result due to the interaction of electromagnetic waves with scatterers are known as the scatter path.

Since a wave carrying the same information in this case travels through different paths they may experience different attenuations. They may also be re- ceived at the receiver in different phases. Due to these two effects these waves may either add constructively or destructively at the receiver.

This causes the received signal power at the receiver to fluctuate. The worst case scenario is the case where the signals that have travelled through different paths add destructively to a point that the total signal power that is received appears to be zero. In such a case a deep fade is said to have occurred. A deep fade is undesirable since it causes detection errors and this significantly increases the bit error rate.

The probability of errors appears to follow a Rayleigh fading distribution in this case and such a channel is consequently referred to as a multipath Rayleigh fading channel. This problem can be best addressed by effecting receive antenna diversity. With receive antenna diversity, there are many receiving antennas. The one that receives the maximum power is chosen. This reduces the probability of errors occurring.

The multipath propagation problem is mainly encountered in wireless communication systems. In wired communication systems this problem does not exist. It is worth noting that the error rate is inversely proportional to the antenna diversity applied. In actual fact if the diversity order is increased and tends to a very large figure, the bit error rate performance of a wireless communication system closely approaches that of a wired communication system.

\section{Simulation results}

\subsection{PN sequence results}

The properties of the PN sequence used are first tested for their randomness properties. Figure 2 shows the

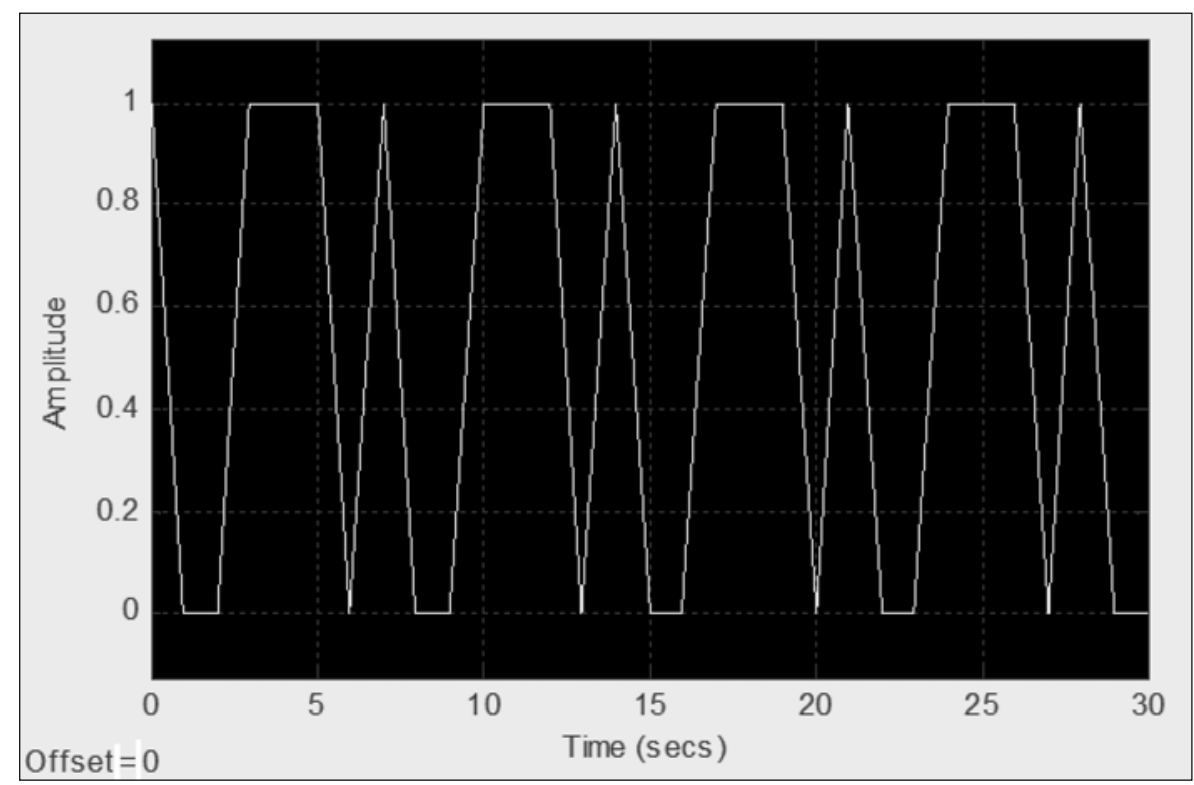

Fig. 2. PN sequence timing diagram 


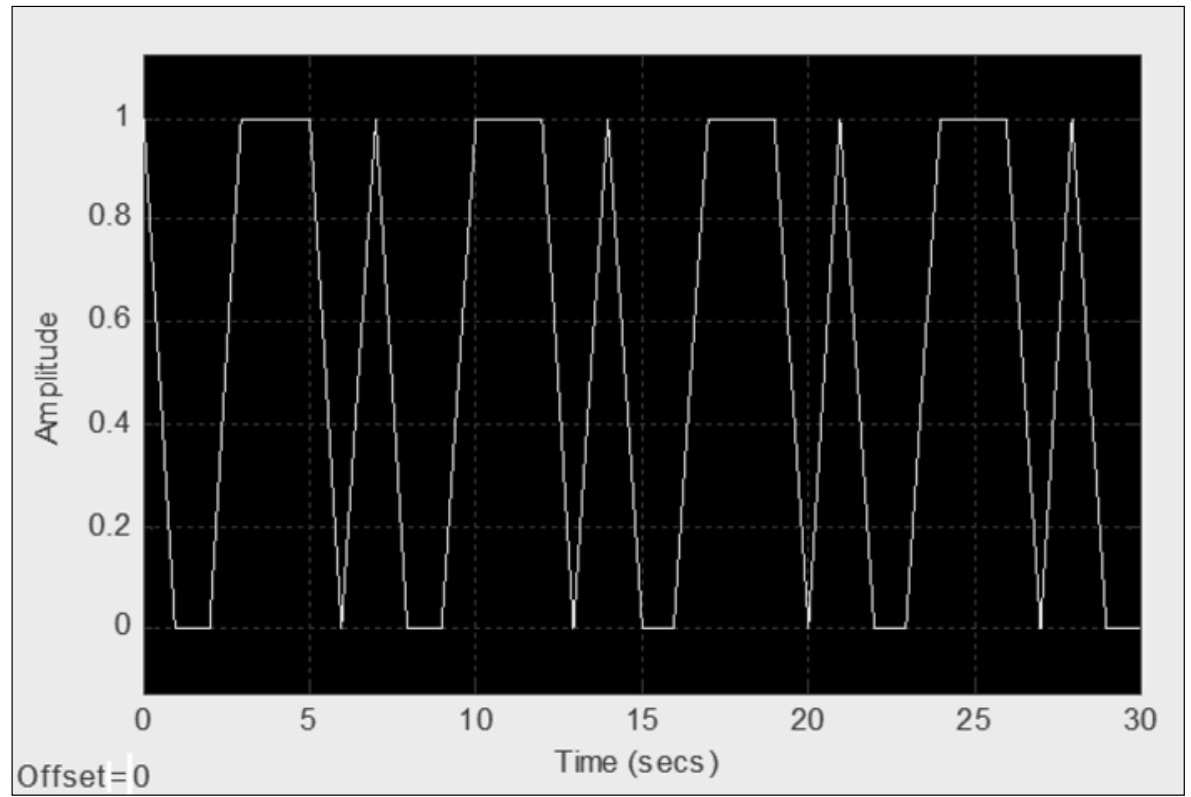

Fig. 3. Correlation between two perfectly aligned PN sequences

timing diagram for a 4-stage LFSR maximal length sequence.

The sample time for the PN sequence is set at $1 \mathrm{sec}$ just for illustrative purposes. For a larger sampling frequency, the basic properties will remain the same. Since the sample time is $1 \mathrm{sec}$, and a maximal length sequence is utilized, the expected PN sequence period is $15 \mathrm{sec}$. From the graph, after a time interval of roughly $15 \mathrm{sec}$ the sequence repeats itself.

The correlation properties of the PN sequences are also tested. From Fig. 2 above, if a sequence similar to the one in Fig. 2 is compared for the number of agreements and disagreements for every $1 \mathrm{sec}$ in- terval for $15 \mathrm{sec}$, the number of agreements would be 15 while the number of disagreements would be zero. This would yield the maximum correlation value. Any shift by a value that is not a multiple of $15 \mathrm{sec}$ would increase the number of disagreements between the un-delayed and delayed version of the PN sequence. This would effectively reduce the correlation between the two signals.

Figure 3 shows the correlation output for two perfectly aligned PN sequences while Fig. 4 shows the correlation output between two PN sequences with a misalignment of $1 \mathrm{sec}$ between each other. Accumulating the output of Fig. 3 over a period of 15

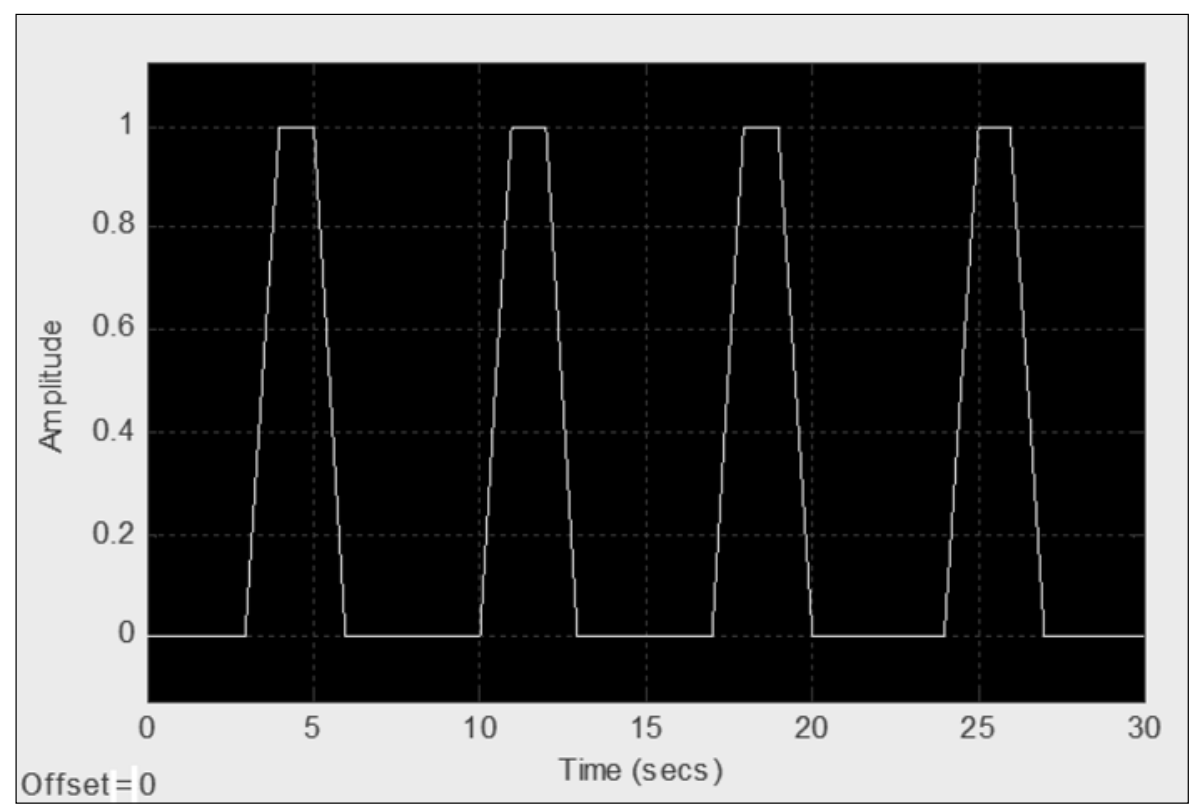

Fig. 4. Correlation between two PN sequences with a misalignment of $1 \mathrm{sec}$ 


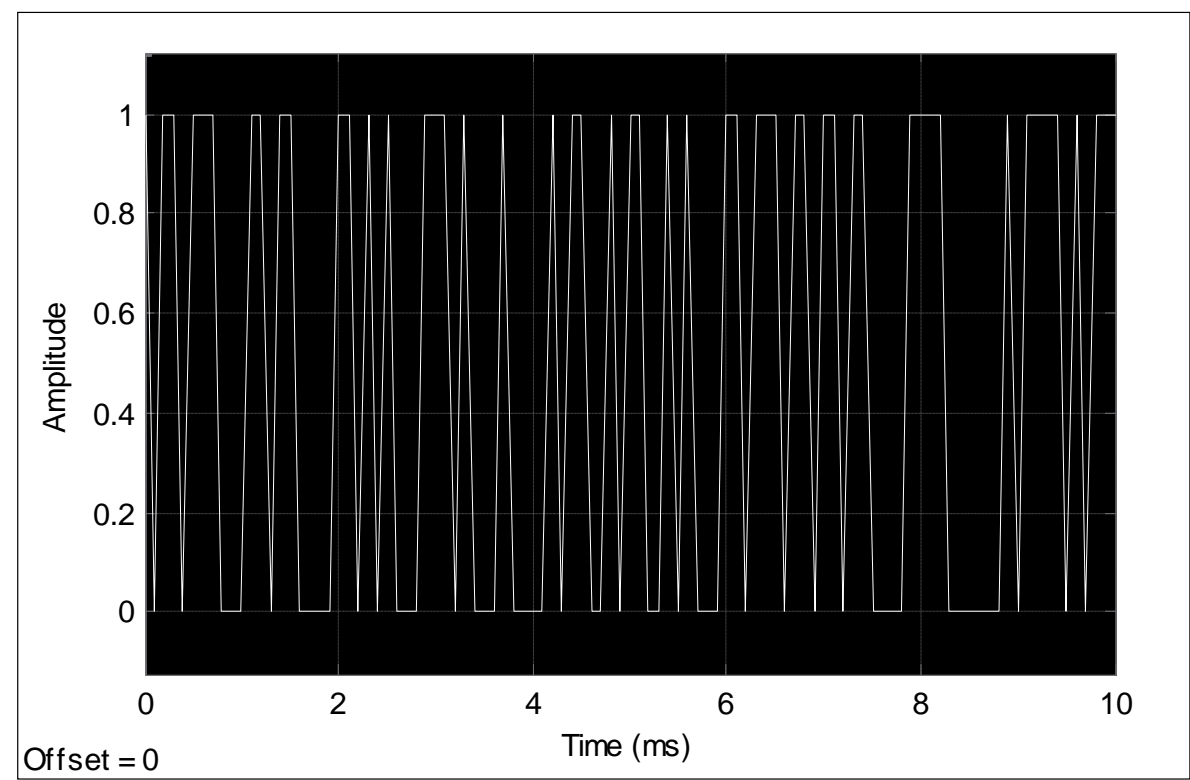

Fig. 5. Transmitted Bernoulli binary signal

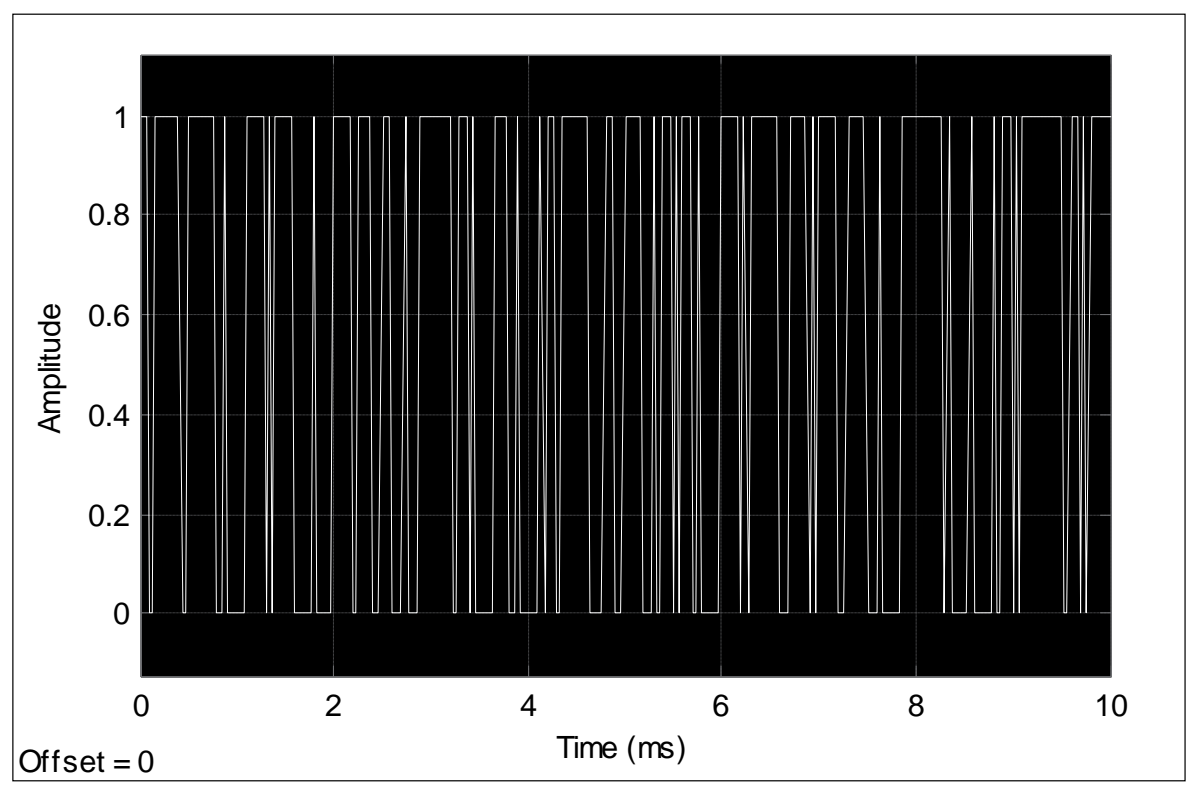

Fig. 6. Received Bernoulli binary signal

sec and doing the same thing for the output in Fig. 4 the output of Fig. 3 yields a larger accumulated value than that of Fig. 4. The output of Fig. 4 represents the situation for all other cyclic shifts. It is therefore evident that the PN sequence has a finite accumulated correlation maximum only when two PN sequences generated by the same generator polynomial are in synchronization.

\subsection{Results for the transmission over multipath Rayleigh channel}

The transmitted Bernoulli binary signal and the received Bernoulli binary signal in the case of a multipath Rayleigh fading channel is shown in Figs 5 and 6 . In this case, the bit error rate is found to be roughly 0.1 . This implies that 1 out of every 10 bits are transmitted in error.

Figures 7-9 show the frequency spectrum of the transmitted signal, the frequency spectrum after frequency hopping and the frequency spectrum after code acquisition, respectively.

\section{Conclusion and discussion}

In this paper, spread spectrum communication systems were studied over Rayleigh channel and the importance of code acquisition in frequency hopping spread spectrum communication systems was observed. The need for code acquisition in FHSS sys- 


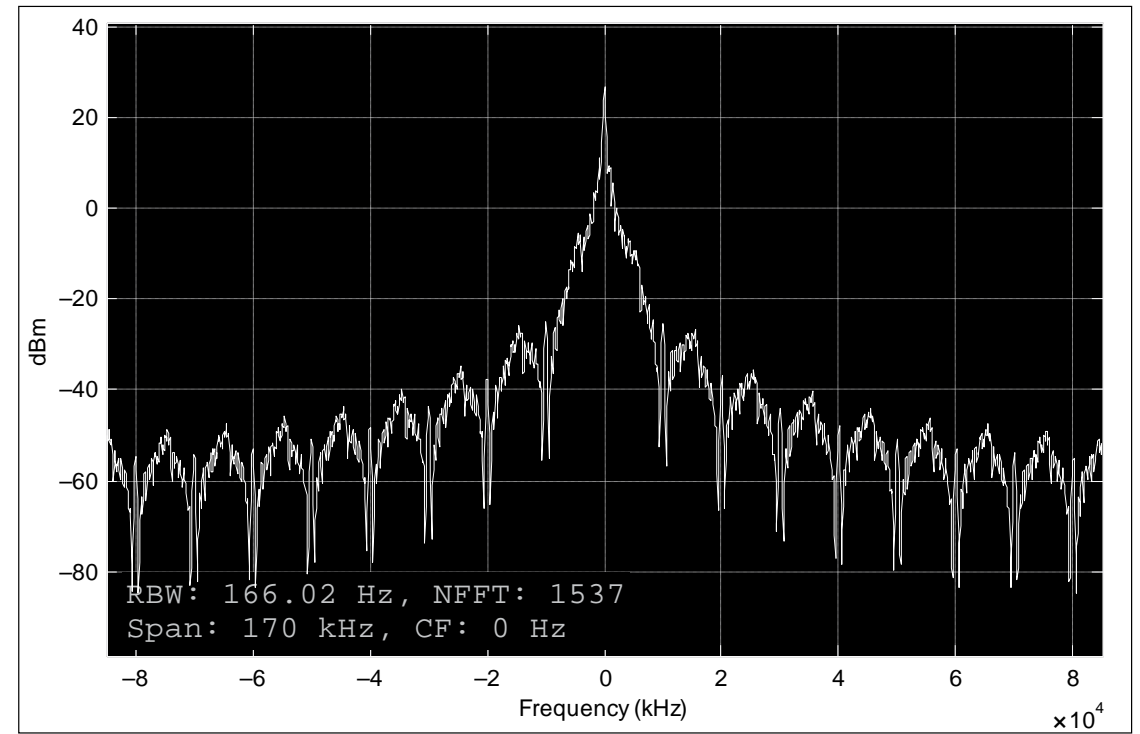

Fig. 7. Frequency spectrum of the transmitted signal after 2-FSK modulation

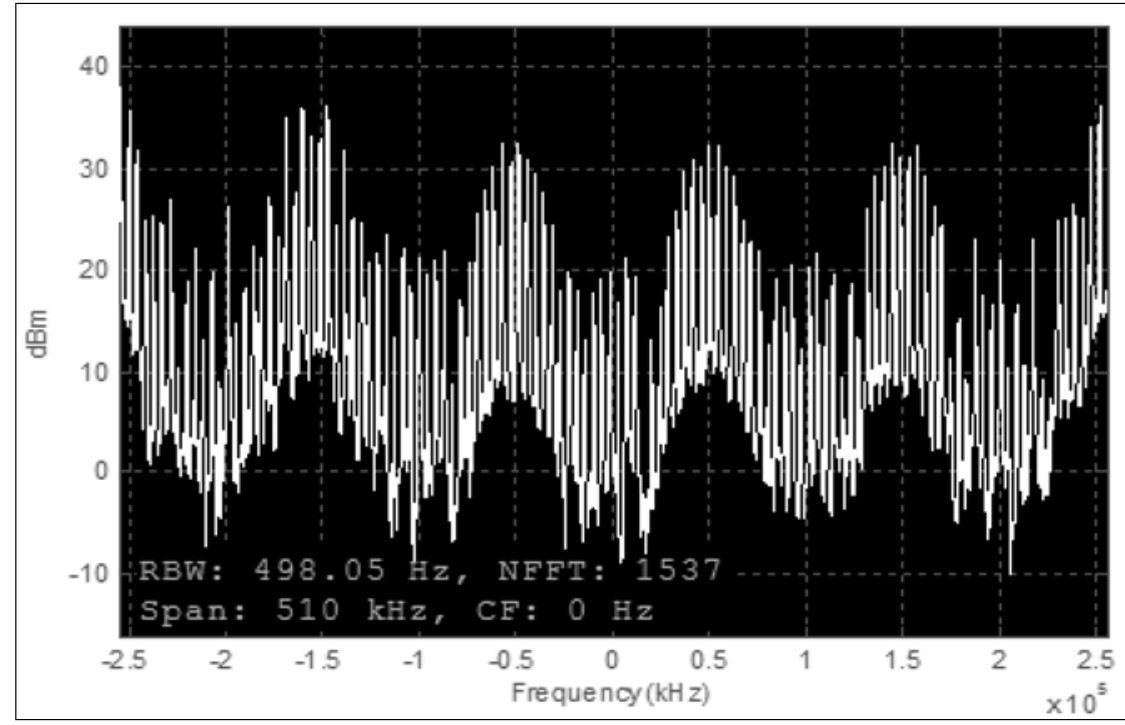

Fig. 8. Frequency spectrum after frequency hopping

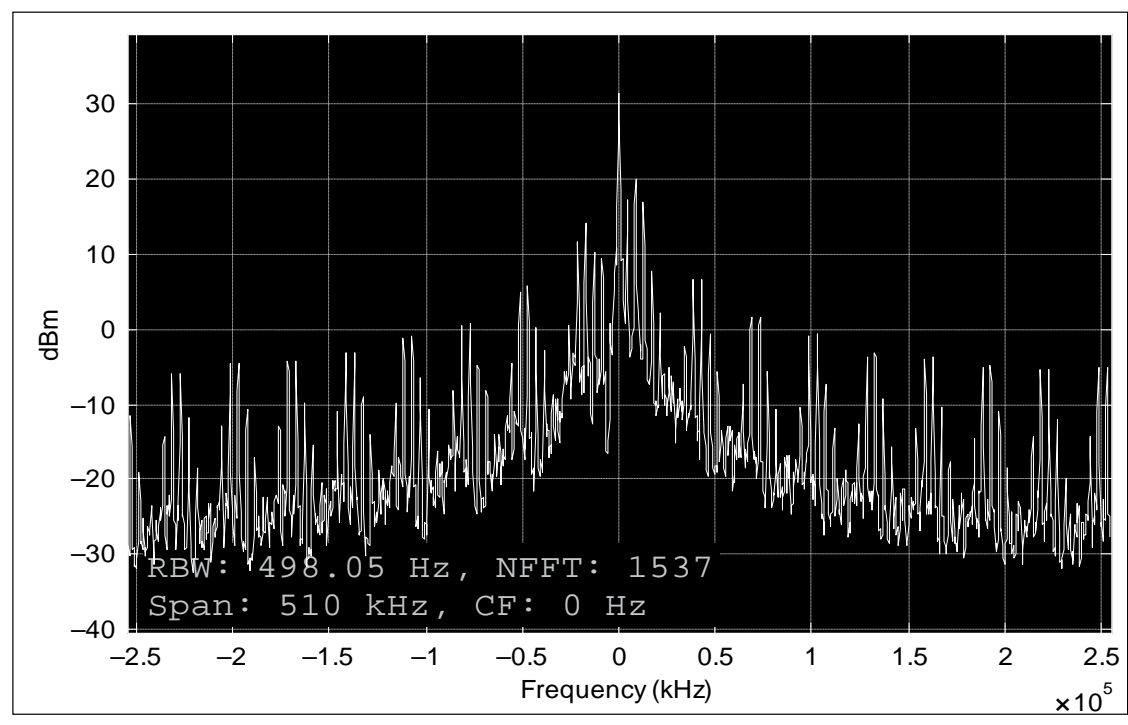

Fig. 9. Frequency spectrum after code acquisition 
tems arises due to clocking instabilities at the receiver and uncertainty in the propagation delay of signals. Code acquisition is a precursor to the fine alignment process of code tracking. Therefore, the results obtained from code acquisition process are not perfect but fine alignment improves the accuracy. A serial search acquisition system was designed and simulated with the performance under a range of channel conditions getting noted. The reason for settling for a serial search acquisition system is informed by its use of fewer components as compared to the matched filter acquisition technique which requires a bank of bandpass filters.

\section{References}

[1] Sklar B. (2009), Digital Communications: Fundamentals And Applications. Second edition, Upper Saddle River, New Jersey: Prentice Hall PTR.
[2] Stallings W. (2007), Data And Computer Communications. Eighth edition, Upper Saddle River, New Jersey: Pearson Education, Inc.

[3] Simon M., Omura J., Scholtz R., Levitt B. (2004), Spread Spectrum Communications Handbook. Electronic edition, McGraw-Hill, Inc.

[4] Torrieri D. (2005), Principles of Spread Spectrum Communication Systems. Springer Science Business Media, Inc.

[5] Viterbi A. (1995), CDMA: Principles of Spread Spectrum Communications. Addison-Wesley Publishing Co.

[6] Zepernick H., Finger A. (2005), Pseudo Random Signal Processing, Theory And Application. John Wiley \& Sons.

[7] Bo Z., Ding S., Elhabian T. (2005), Study on fast acquisition of hybrid DS/FHSS. Chinese Journal of Aeronautics, 18(2), 161-165.

[8] Zhao X., Quan H., Cui P. (2018), Research on Synchronization Technology of Frequency Hopping Communication System. In: 6th International Conference on Computer-Aided Design, Manufacturing, Modeling and Simulation (CDMMS), Busan, South Korea. 